\title{
Electron pair emission from surfaces: Photon versus electron excitation
}

\author{
F. O. Schumann $\odot^{*}$ and J. Kirschner \\ Max Planck Institut für Mikrostrukturphysik, Weinberg 2, 06120 Halle, Germany
}

(Received 9 December 2020; revised 5 February 2021; accepted 16 February 2021; published 24 February 2021)

\begin{abstract}
We have studied the electron pair emission process from a $\mathrm{Cu}(111)$ surface excited by a primary electron or photon beam. Although the photon energy was $30 \mathrm{eV}$ the primary electron beam had an energy of $25 \mathrm{eV}$. We will discuss the energy and momentum distributions. The experiments with the electron beam demonstrate that the contributions from the Shockley surface state can be clearly identified. This is not the case for the work with photons which we ascribe to the low spectral weight of the contribution from two Shockley state electrons compared to two $3 d$ electrons. The momentum distributions reveal a close similarity between the two types of excitation despite the fact that the microscopic origin of the pair emission is very different. There is a distinctive energy dependence of these curves which we describe by an empirical three-parameter polynomial. The numerical values of these parameters are close for both pathways. Additional measurements with different primary electron energies reveals the importance of the initial state on the momentum distributions.
\end{abstract}

DOI: 10.1103/PhysRevB.103.085435

\section{INTRODUCTION}

Electron spectroscopy is an important tool to study the properties of matter. Specifically, angle-resolved photoemission where the kinetic-energy distribution of photoemitted electrons is measured for a fixed emission angle has matured into a powerful tool. In particular, the energy position of intensity peaks in the spectrum provides access to the electronic band structure of solids. This is intimately related to the dispersion $E(k)$ of electronic structure calculations. The current status in terms of energy and angular resolution allows to identify features which arise due to many-body effects, e.g., electron-electron or electron-phonon coupling. The discussion of these results is usually performed within a single-particle description.

More direct insights into the electron-electron interaction is possible if one performs experiments in which an electron pair is detected upon single-particle excitation. We will in the following discuss pair emission triggered by primary electron impact or single-photon absorption. The processes are usually referred to as $(e, 2 e)$ and double photoemission (DPE).

An important prediction of theory was that for both processes the emission direction of one electron defines a region of low intensity of the second electron [1,2]. This depletion zone is intimately related to the concept of the exchangecorrelation hole. The existence of this concept in $(e, 2 e)$ and DPE was experimentally verified [3-10].

\footnotetext{
*schumann@mpi-halle.de
}

Published by the American Physical Society under the terms of the Creative Commons Attribution 4.0 International license. Further distribution of this work must maintain attribution to the author(s) and the published article's title, journal citation, and DOI. Open access publication funded by the Max Planck Society.
The $\mathrm{Cu}(111)$ surface exhibits a prominent surface state known as the Shockley surface state in the center of the Brillouin zone. This is a two-dimensional (2D) electronic state which displays a quadratic dispersion. The occupancy is about $1 / 30$ electron per surface atom [11]. The Shockley surface state has been widely studied in angle-resolved photoemission [12-18].

The 2D nature of this state brought it into the center of a variety of theoretical and experimental $(e, 2 e)$ or DPE studies because it allows to investigate the effects of electronelectron correlation [7,19-27]. Almost all theoretical works either discussed the $(e, 2 e)$ or the DPE pathway. Only in one work the two processes have been calculated within the same framework and equivalent kinematics [19]. On the experimental side only one published work exists which describes the $(e, 2 e)$ and DPE process from a $\mathrm{W}(100)$ surface with comparable kinematics [28].

In this paper we discuss 2D-energy and momentum distributions from a $\mathrm{Cu}(111)$ surface in $(e, 2 e)$ and DPE. The 2D-energy distributions for $(e, 2 e)$ display sharp structures which can be related to the contribution of the Shockley surface state as reported previously [23-25]. This identification is not possible in DPE, which is a consequence of the low spectral weight compared to the emission of two $3 d$ electrons. Imposing a geometrical constraint has a dramatic effect on the 2D-energy spectra in $(e, 2 e)$. The contribution of equal energy electrons is strongly suppressed if the two outgoing electrons are emitted close to the normal. This effect is also present in the DPE process but much weaker.

The momentum distributions for DPE and $(e, 2 e)$ display a reduced intensity for electron pairs whose trajectories include small emission angles as discussed in the context of the exchange-correlation hole $[3-6,8,9]$. This is an important concept of solid-state theory [29-31]. Here we provide a more detailed analysis of the line shape. 


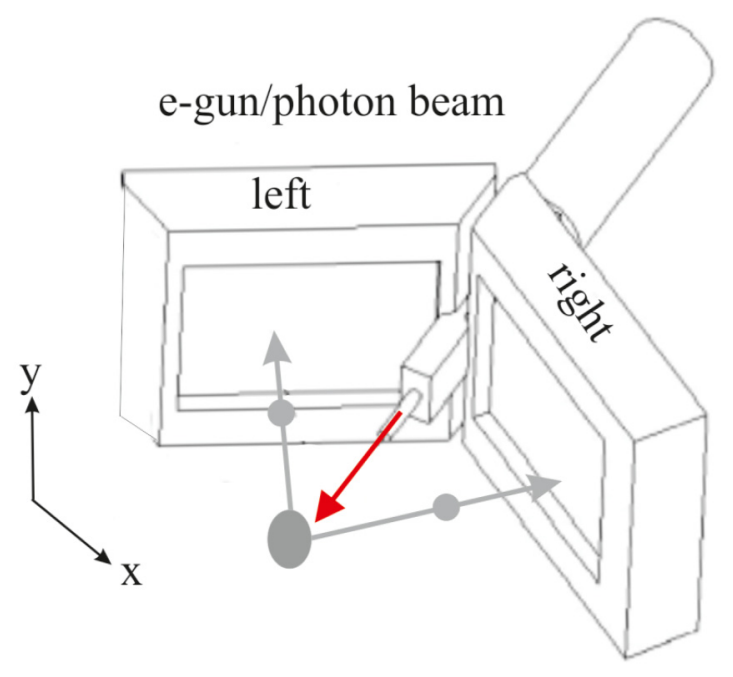

FIG. 1. Schematic of the TOF spectrometer. It consists of two channel plates labeled left and right, respectively. This allows to record the impact position of the emitted electrons. The excitation source is a pulsed electron gun or a photon beam.

Despite the difference in the microscopic process leading to electron pair emission the momentum distributions for $(e, 2 e)$ and DPE are very similar. In both cases the shape displays a distinct energy dependence which can be described by a polynomial with three parameters. Furthermore, the numerical values of these parameters are very similar for DPE and $(e, 2 e)$. Additional $(e, 2 e)$ measurements with a different primary energy reveal that the momentum profile is largely determined by the initial state. This, in turn, emphasizes the sensitivity of electron pair emission on the correlation of the initial state.

\section{EXPERIMENT}

In Fig. 1 we provide a schematic of our time-of-flight (TOF) setup which we described previously $[6,8]$. Two delay line detectors facilitate the determination of the impact positions of the electrons. The angular acceptance of each detector amounts to $\pm 27^{\circ}$ in one direction whereas perpendicular it is $\pm 22^{\circ}$. We will label the detectors as left and right, respectively. Each detector captures a solid angle of approximately $11 \%$ of the half sphere. The instrument is actually equipped with three microchannel plates (MCPs), but for normal incidence of photons or electrons, the sample essentially blocks the third detector. Therefore, we operated with two MCP detectors. For $(e, 2 e)$ experiments we use a pulsed electron beam with $2-\mathrm{MHz}$ repetition rate. From the impact position the emission angles and the flight path are known. The electron energies are determined via the flight times. A coincidence circuit ensures that only one pair can be detected for each incoming electron or photon pulse. The DPE experiments were performed in the single bunch mode at the CP-NIM beamline of the BESSY II storage ring at the Helmholtz-Zentrum Berlin [32]. The time between subsequent bunches is $800 \mathrm{~ns}$ which amounts to a repetition rate of $1.125 \mathrm{MHz}$. This beamline provides circurlar polarized light, we added the spectra for the two helicities. We did not observe any dependence on the polarization as it is expected for our geometry [33,34]. We selected a photon energy of $30 \mathrm{eV}$, and the incoming beam was parallel to the sample normal. The spectrometer is part of an ultrahigh-vacuum system equipped with standard surface science tools, such as Auger electron spectroscopy and low-energy electron diffraction (LEED). The preparation of the $\mathrm{Cu}(111)$ sample followed standard procedures of $\mathrm{Ar}^{+}$ sputtering and annealing up to $800 \mathrm{~K}$. This resulted in a clean and well-ordered sample surface. The quality of the sample was further confirmed by the observation of spectroscopic features due to the Shockley surface state in $(e, 2 e)$ spectra. All experiments were performed at room temperature, more details can be found elsewhere [6,24]. For all experiments the [1110] direction of the $\mathrm{Cu}(111)$ surface was aligned along the $x$ axis, see Fig. 1. Each coincident event is stored in a list and is characterized by six parameters, namely, the individual energies and the two components of the in-plane momentum of each electron. The latter uniquely define the emission direction of each electron. The total time resolution is approximately $1.1 \mathrm{~ns}$ for $(e, 2 e)$ and $0.5 \mathrm{~ns}$ for DPE. This will lead to an energy-dependent energy resolution, which is 0.62 and $0.29 \mathrm{eV}$ for $13 \mathrm{eV}$ electrons, respectively.

\section{RESULTS}

\section{A. Kinematics}

Let us formulate energy and in-plane momentum conservation for the $(e, 2 e)$ and DPE process [10,35,36]. In an $(e, 2 e)$ process a primary electron with kinetic-energy $E_{p}$ hits a surface and ejects a valence electron with binding energy $E_{v b}$. The emitted electrons posses the kinetic-energies $E_{1}$ and $E_{2}$, respectively. In the following we refer these energies to the vacuum level of the sample. The work function of the $\mathrm{Cu}(111)$ surface is $\phi=4.88 \mathrm{eV}$, and we can finally write for the energy balance,

$$
E_{p}+E_{v b}=E_{1}+E_{2}+\phi=E_{\text {sum }}^{e 2 e}+\phi .
$$

The energy sum of the emitted pair attains the largest value of $E_{p}-\phi$ if the valence electron stems from the Fermi-level $E_{F}$. In a straightforward manner we can formulate energy conservation for DPE as

$$
h v+E_{v b 1}+E_{v b 2}=E_{1}+E_{2}+2 \phi=E_{\text {sum }}^{D P E}+2 \phi .
$$

Since two valence electrons are ejected the work function has been accounted twice. Conceptually the work function to emit a pair is not equal to twice the work function because of the correlation energy. A recent study showed that the deviation must be smaller than $200 \mathrm{meV}$ as far as DPE from valence states are concerned [37].

Similarly we formulate for the in-plane momentum conservation in $(e, 2 e)$,

$$
\boldsymbol{k}_{p, \|}+\boldsymbol{k}_{v b, \|}=\boldsymbol{k}_{1, \|}+\boldsymbol{k}_{2, \|}+\boldsymbol{g}_{\|}=\boldsymbol{k}_{\text {sum }, \|}+\boldsymbol{g}_{\|} .
$$

The in-plane momentum of the primary beam is given by $\boldsymbol{k}_{p}$ whereas $\boldsymbol{g}$ is the reciprocal lattice vector. This formulation can be easily extended for DPE, and we obtain

$$
\boldsymbol{k}_{v b 1, \|}+\boldsymbol{k}_{v b 2, \|}=\boldsymbol{k}_{1, \|}+\boldsymbol{k}_{2, \|}+\boldsymbol{g}_{\|}=\boldsymbol{k}_{\text {sum }, \|}+\boldsymbol{g}_{\|} .
$$

There are some key differences between the two types of processes within the current theoretical description. After the 
excitation the sample has lost one electron in $(e, 2 e)$ whereas in DPE two electron are removed. Therefore, theory considers the initial state in $(e, 2 e)$ as a two-electron state comprising the incoming primary electron and a valence electron $[1,19,35,38-40]$. In DPE the initial state is given by two valence electrons $[2,41]$. In both instances the initial state is an antisymmetrized product of single-particle wave functions. This means the electrons are considered to be noninteracting. As a final state one assumes in both cases a pair of timereversed LEED states. In order to incorporate the effect of the electron-electron interaction one introduces a correlation factor $f_{c}$ [19]. This entity depends on the position and momentum difference of two electrons. This approach ensures that the final state is not an antisymmetrized product of single electron states but a correlated wave function. The function $f_{c}$ is computed by considering two plane waves interacting via a screened Coulomb potential labeled as $U$. In this way the final state possesses correlation effects emanating from the ground state of the sample. The calculation of electron pair spectra requires to evaluate a matrix element between an initial and a final state. The operator is in the case of $(e, 2 e)$ the screened Coulomb interaction $U$ whereas for DPE it is the dipole operator $D_{1}+D_{2}[2,19,35,36,40,41]$. Let us formulate the matrix elements in a shorthand notation for $(e, 2 e)$ as follows:

$$
\left\langle i|U| f_{c}, 1,2\right\rangle .
$$

The screened Coulomb interaction $U$ is the same as used for the calculation of the correlation factor $f_{c}$. With $\left|f_{c}, 1,2\right\rangle$ we identify the two time-reversed LEED states which are correlated by means of the correlation factor. The initial state for $(e, 2 e)$ is then $\langle i|$ given by a valence state and incoming LEED state. Similarly we formulate for the DPE matrix element,

$$
\left\langle i\left|D_{1}+D_{2}\right| f_{c}, 1,2\right\rangle \text {. }
$$

The final state, as discussed before, is the same for $(e, 2 e)$ and DPE. The dipole operator is given by the term $D_{1}+D_{2}$. It has the structure of a sum of two dipole operators. This fact has the important implication that the description of both initial and final states as antisymmetrized single electron wave functions will yield zero DPE intensity [36,42]. It is mandatory to include, at least, for either the initial or the final state a correlated two-electron wave function. The introduction of the correlation factor $f_{c}$ ensures that the final state fulfills this requirement.

Additionally there can be only intensity if there exist initial states consistent with the above conservation laws. Therefore, discussing the band structure is a first approximation of the expected spectral features.

Theory can pick a particular initial state and calculate the pair spectrum. The experimentalist has to proceed differently because one has no control which initial state is excited. However, energy and momentum conservation allow to infer this information. An emitted pair is characterized by the kinetic energies and in-plane momenta of the two emitted electrons. In the case of $(e, 2 e)$ Eqs. (1) and (3) uniquely define the valence state. This allows to select the initial state of interest in a postexperiment analysis, e.g., the Shockley surface state [24]. This is very different for DPE because the left-hand side of Eqs. (2) and (4) has two entries from the band structure.

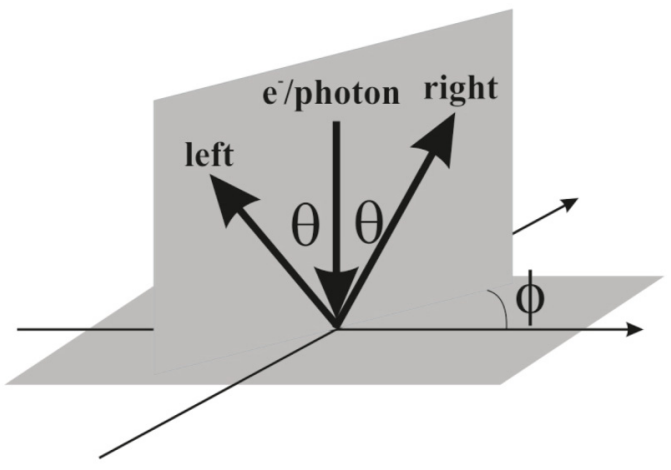

FIG. 2. In a coplanar geometry a normal incident primary beam of photons or electrons hits the sample. Two electrons have an emission angle $\theta$ with respect to the surface normal. Both electrons propagate within the same plane but opposite directions. This plane is perpendicular to the surface and has an azimuthal orientation defined by $\phi$.

Therefore, each combination which gives the same sum of binding energy and in-plane momentum is compatible with the experimental determined values.

If we assume a constant matrix element then DPE requires a self-convolution of the band structure. We performed this evaluation for $\mathrm{Cu}(111)$, and more details can be found in the Supplemental Material [43].

\section{B. Two-dimensional-energy distributions}

We recall the emission geometry assumed in a variety of theoretical pair emission studies, see Fig. 2 [1,19,38]. An incoming beam of primary electrons or photons propagates along the surface normal. Two electrons are ejected with the same angle $\Theta$ with respect to the surface normal. They propagate along trajectories which are contained within a plane perpendicular to the surface plane. The azimuthal orientation of this plane is described by the angle $\phi$.

The calculation then proceeds mainly along two routes. First, the evaluation is performed for fixed angles $\phi$ and $\Theta$ but variable emission energies. Second, for a given angle $\phi$ and equal emission energies the intensity as a function of the angle $\Theta$ is plotted. In this case the in-plane sum momentum is exactly zero. In order to select the experimental data in an analogous way we proceed as follows. In Fig. 3 we plot the coincidence intensity of all events. As coordinates we use the normalized components of the in-plane momentum $k_{x} / k$ and $k_{y} / k$, respectively. The term $k$ is the length of the momentum vector using all three components. We impose a geometrical constraint on the emission directions defined by the pair of arcs on the left and right detectors. The mean radii of the arcs is given by $\Delta$. The separation of the arcs on the left and right is 0.2 , which is governed by the need to capture sufficient intensity.

We proceed and present 2D-energy distribution for DPE under various constraints $\Delta$, see Fig. 4 . The choice of $\Delta=$ 0.3 (0.7) selects events which are in the minimum (maximum) of the momentum distributions as we will discuss below. We added to both panels a black diagonal line which indicates the position of $E_{\text {sum }}^{\max }$. In Fig. 4(a) we have added an ellipse 


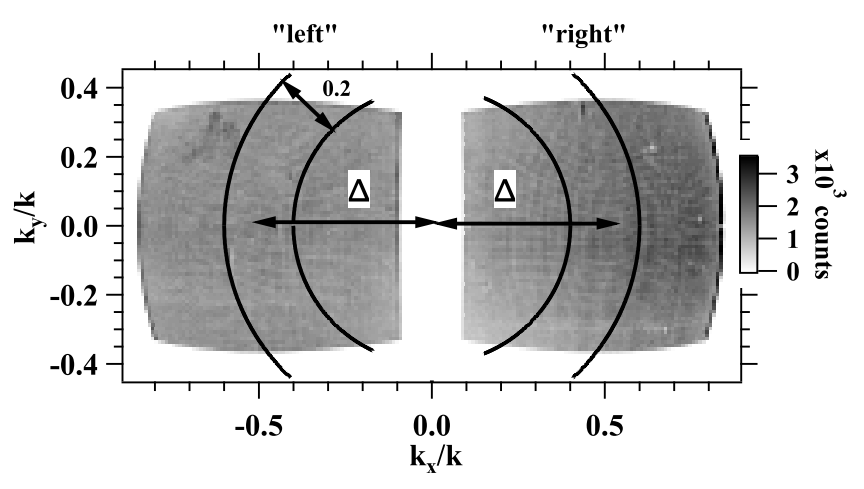

FIG. 3. Intensity distribution of all coincidence events as a function of the normalized in-plane momentum components $k_{x} / k$ and $k_{y} / k$. The $x$ axis is along the [110] direction. A pair of arcs defines a region of interest for which we compute $2 \mathrm{D}$-energy distributions, see below.

to describe an energy selection employed for momentum distributions, see below. Our selection focuses on those initial states where the in-plane momentum is close to zero. The DPE data display a smooth increase in the intensity if the electron energies become smaller. There are no obvious intensity features, and near the $E_{\text {sum }}^{\max }$ line the intensity is low. The DPE data reveal only a weak dependence on the parameter $\Delta$. For $\Delta=0.7$ the intensity parallel to the $E_{\mathrm{sum}}^{\max }$ line is constant. For $\Delta=0.3$ there is a weak preference for unequal energies.

In contrast to the DPE data, the results of the $(e, 2 e)$ study reveal a strong dependence on the parameter $\Delta$, see Fig. 5 . For $\Delta=0.3$ the intensity near $E_{\text {sum }}^{\max }$ has almost vanished, only for very unequal energies intensity is seen. In general, there is a reduction of intensity for equal energies except for low energies of both electrons. This situation changes significantly for $\Delta=0.7$, now intensity for equal energies has returned. The contribution from the Shockley surface state is confined to a smaller energy range along the diagonal direction. This is a straightforward consequence of the kinematics for the Shockley surface state, namely, the localization near the center of the Brillouin zone. The spectrometer is able to identify the contribution of the Shockley surface state in $(e, 2 e)$ but not in DPE. This is related to the expected small spectral weight as discussed in the Supplemental Material [43]. The key result is that available $2 e$-DOS for Shockley pair electrons in DPE is about three orders of magnitude smaller than for bulk states, see Fig. 6.

\section{Momentum contributions}

Coming back to the geometry of Fig. 2 we want to select equal emission energies and plot the intensity as a function of the in-plane momentum. More precisely we will use the normalized in-plane momentum which facilitates comparison for different emission energies. First, we execute an energy selection as indicated by the ellipse in Fig. 4(a). In this example the ellipse is centered at $E_{\text {left }}=E_{\text {right }}=10 \mathrm{eV}$. The dimension ensures that enough counts are available for presentation. We keep the size of the ellipse fixed, the width parallel to the $E_{\text {sum }}^{\max }$ line is $5.7 \mathrm{eV}$ whereas it is $2.8 \mathrm{eV}$ perpendicular to it.

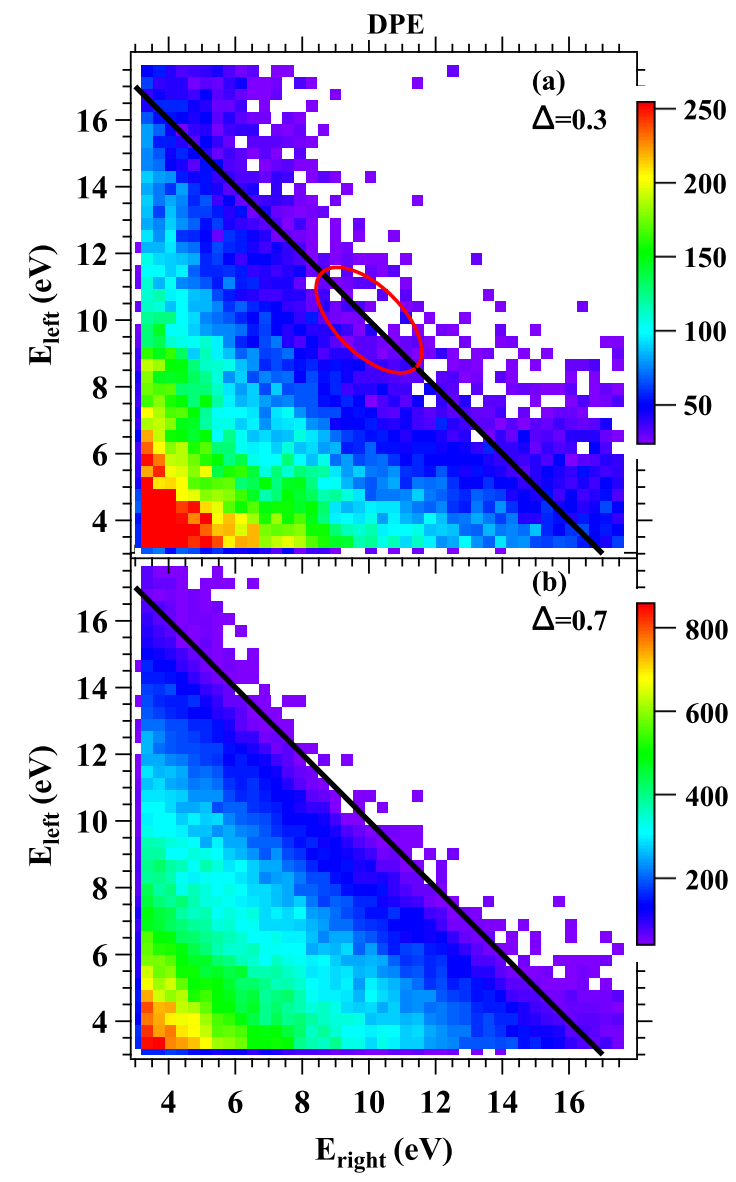

FIG. 4. In panel (a) we show the 2D-energy distribution for the DPE data obtained with $h v=30 \mathrm{eV}$. Panel (a) is obtained with the constraint $\Delta=0.3$, whereas panel (b) is the result for $\Delta=0.7$. The black diagonal represents the $E_{\text {sum }}^{\max }$ line. The white ellipse indicates the energy window used for the selection of events for momentum plots.

In Fig. 7 (a) we show the in-plane momentum distribution for DPE with $h v=30 \mathrm{eV}$ and $E_{\text {left }}=E_{\text {right }}=9 \mathrm{eV}$. There is an intensity variation such that for small values of $\left|k_{x} / k\right|$ the level is lowest and progressively increases as one moves towards the detector edges on the left and right, respectively. The intensity is highest near the edge of the detectors.

Second, we use an additional constraint and consider only those events for which the sum momentum $\left|k_{\text {sum }}\right| \leqslant 0.2 \AA^{-1}$. The result is presented in Fig. 7(b). It is apparent that there is a depletion zone which we brought into context with the concept of the exchange-correlation hole $[1-6,8,9,19]$. The observation of the full extension of the depletion zone requires a large angular acceptance which was possible with the current apparatus if all three detectors were used $[5,6]$. As stated above, the work in normal incidence restricted the use to two detectors. This means the intensity maxima at the left (right) side of Fig. 7 should not be regarded as a measure of the extension of the depletion zone.

A further analysis will be performed on line scans of the 2D-momentum distributions. For this we integrate the intensity within the area bounded by the pair of dashed lines in Fig. 7(b). This sampling is needed to obtain enough counts. 


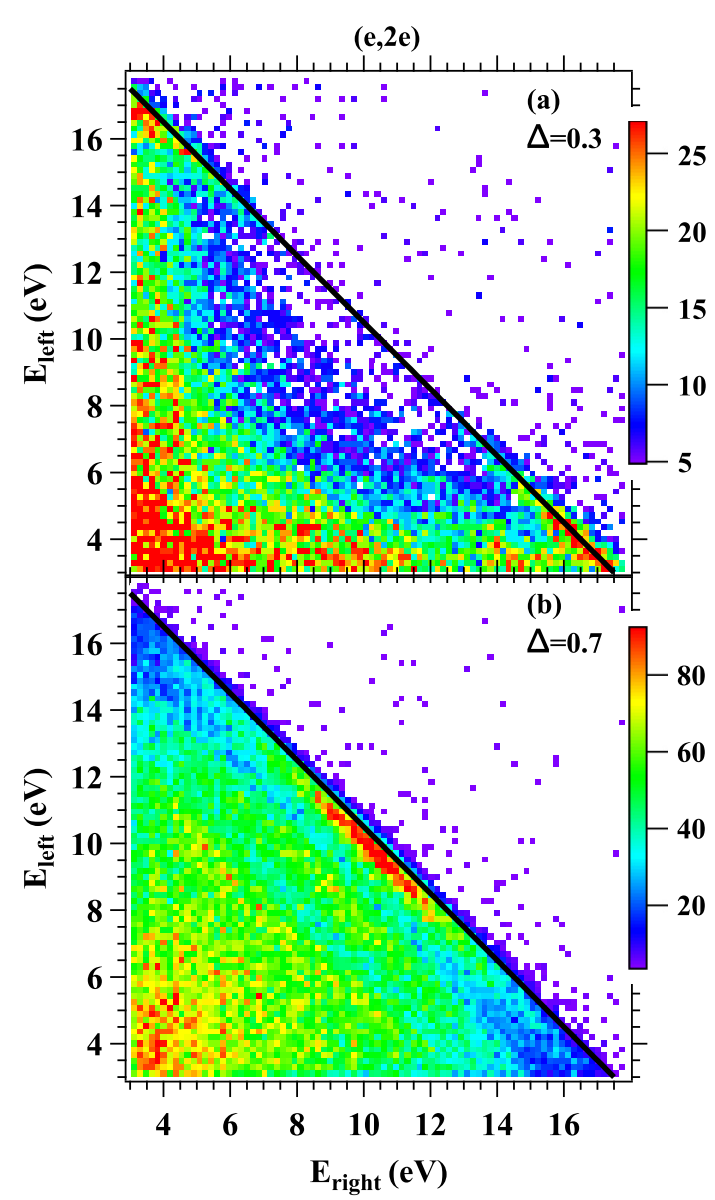

FIG. 5. In panel (a) we show the 2D-energy distribution for the $(e, 2 e)$ data obtained with $E_{p}=25 \mathrm{eV}$. Panel (a) is obtained with the constraint $\Delta=0.3$, whereas panel (b) is the result for $\Delta=0.7$. The black diagonal represents the $E_{\text {sum }}^{\max }$ line.

The resulting line profiles are presented in Fig. 8. In those we compare the DPE and $(e, 2 e)$ data for the same energy and momentum constraint $\left|k_{\text {sum }}\right| \leqslant 0.2 \AA^{-1}$. In the middle of the line scan near $k_{x} / k=0$ there is no intensity simply because there is no detector area. Likewise the rapid falloff at the extreme values for $k_{x} / k$ is caused by the finite detector area. This has the effect that the line scans display maxima near these positions. As we can see from Fig. 8 the momentum distributions for DPE and $(e, 2 e)$ are rather similar. A noticeable difference between the DPE and the $(e, 2 e)$ data concerns the behavior near $k_{x} / k=0$. For the latter the intensity is lower than for DPE. Although we cannot quantify it, the DPE data have been obtained with a higher background of random coincidences. From a selection of events which are above the $E_{\text {sum }}^{\max }$ line we know that they will contribute an almost constant intensity to the profile lines. Therefore, we ascribe a portion of larger intensity near $k_{x} / k=0$ in DPE from this effect. It is apparent that the line profiles for $5 \mathrm{eV}$ are significantly different from those for $9 \mathrm{eV}$. In simple terms we may describe the 9-eV data as displaying a $\mathrm{U}$ shape, whereas the 5-eV data resemble more a V shape. By this is meant that for small $\left|k_{x} / k\right|$ values the $9-\mathrm{eV}$ data maintain a low intensity and increase more rapidly upon approaching the extreme $\left|k_{x} / k\right|$ values at

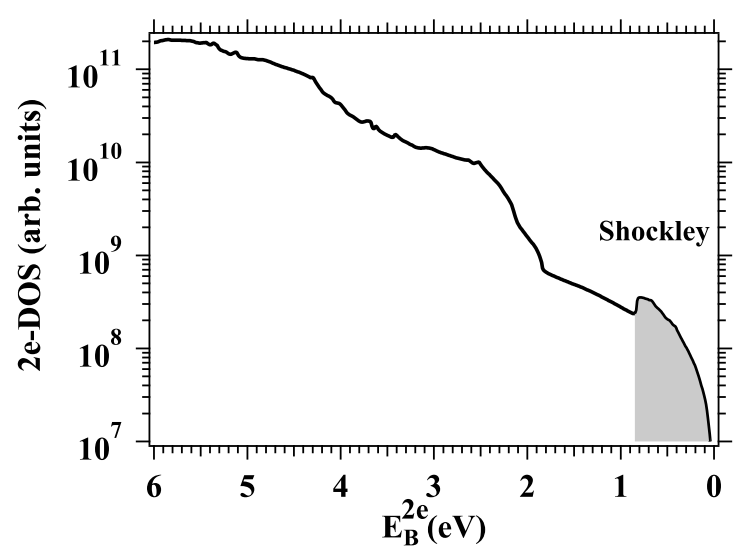

FIG. 6. Available initial states as a function of the two-electron binding energy within a momentum range. The $2 e$-DOS is displayed on a logarithmic scale. The region covered by the Shockley electron pair is marked by the gray area. More details are presented in the Supplemental Material [43].

the detector edges. This obvious qualitative behavior warrants a quantitative analysis. We want to describe the line profiles in Fig. 8 by a simple function. As far as the theory is concerned we are not aware of a functional expression. The symmetry of the emission geometry suggests a polynomial with even powers only. We attempt a description with three parameters, and we write

$$
I(x)=a_{0}+a_{2} x^{2}+a_{4} x^{4} .
$$

The quantity $x$ equals $k_{x} / k$ which is the directional cosine. The fit curve has been added to the profiles in Fig. 8 as red curves. We limited the analysis to $\left|k_{x} / k\right|$ values smaller than 0.7 .

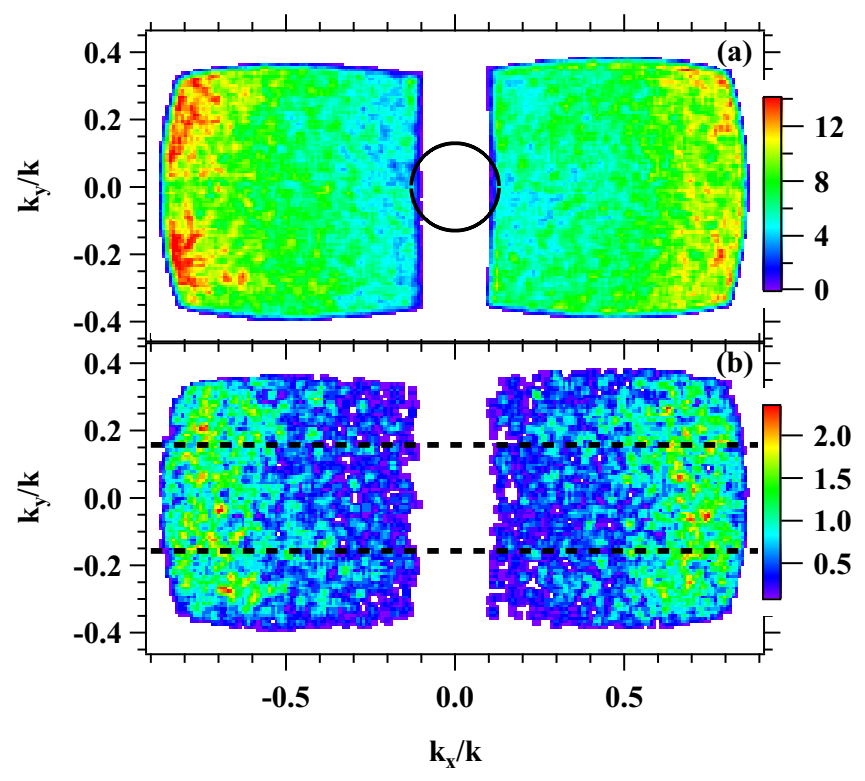

FIG. 7. Two-dimensional-momentum plot from DPE measurements from a $\mathrm{Cu}(111)$ surface with $h v=30 \mathrm{eV}$. The central energies for the two electrons are $9 \mathrm{eV}$. The solid circle in (a) indicates the sum momentum constraint which leads to the plots in panel (b). The dashed lines in panel (b) indicate the integration range in order to get sufficient intensity for the line profiles. 
$9 \mathrm{eV}$

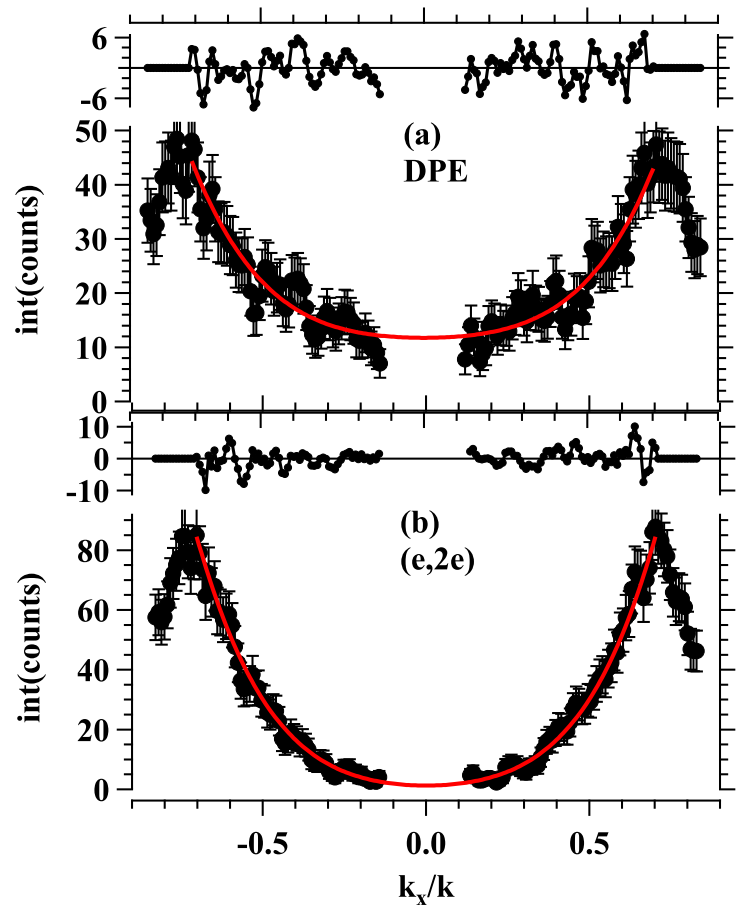

$5 \mathrm{eV}$

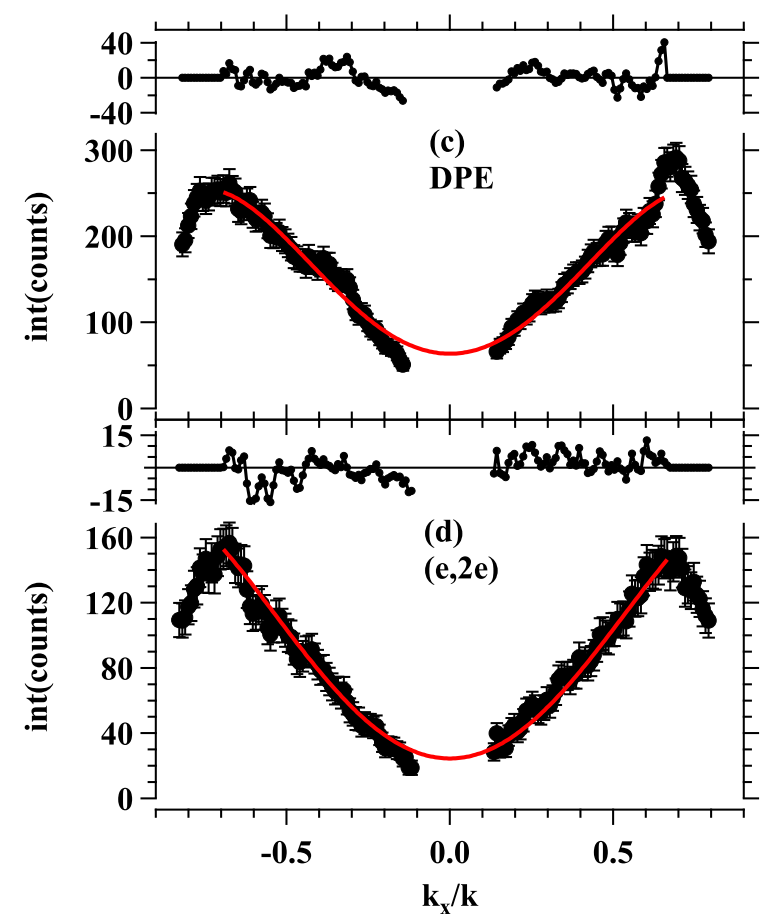

FIG. 8. DPE and $(e, 2 e)$ momentum line scans under the constraint of the sum momentum $\left|k_{\text {sum }}\right| \leqslant 0.2 \AA^{-1}$. Panels (a) and (b) are for $E=9 \mathrm{eV}$ whereas in (c) and (d) the energy of the electrons is $E=5 \mathrm{eV}$. The red curve is the polynomial fit as discussed in the text. The residual is plotted on top of each graph.

This ensures that one is sufficiently away from the intensity maximum which is not a true maximum but a consequence of reaching the detector edges. Additionally we plot the residual in each panel. Those show that the simple function we employed captures the essentials and systematic deviations are rather small.

Even if two line profiles are the same, the fit parameters $a_{0}, a_{2}$, and $a_{4}$ will vary if the intensity levels are different. For comparison we normalize them. First, we determine the fit parameters using the experimental curve. Second, we calculate the intensity of the fit at the minimum/maximum value for $x$ within the window of the fit. Now we divide the fit parameters and their variance by this intensity value. In other words, we normalized the fit curve such that the maximum value of the profile is 1 .

The energy dependence of the normalized parameters $a_{0}, a_{2}$, and $a_{4}$ are shown in Fig. 9. The black and red data points concern the DPE and $(e, 2 e)$ experiment which are kinematically equivalent. The primary energy of the $(e, 2 e)$ experiment is then the photon energy minus the work function. An additional $(e, 2 e)$ experiment with a primary energy of $29 \mathrm{eV}$ has been analyzed, and the results are given by the blue data points. Let us focus on the black and red data points first.

The contribution of the constant term $a_{0}$ is shown in Fig. 9(a). The main difference between the data for $E_{p}=$ $25 \mathrm{eV}$ and DPE is the increase in the latter as a function of the energy. This relatively large value for the DPE data is likely related to the larger contribution of random coincidences for the DPE experiment compared to the $(e, 2 e)$ study. The behavior of random coincidences can be accessed by evaluating the profile for energies well above $E_{\text {sum }}^{\max }$. This leads to an almost constant intensity distribution as a function of $k_{x} / k$.

In Fig. 9(b) we present the behavior of the quadratic term $a_{2}$. For both the DPE and the $(e, 2 e)$ experiment with $E_{p}=$ $25 \mathrm{eV}$ a rather similar behavior is seen. For increasing energy an almost linear decrease is visible, and for $10 \mathrm{eV} a_{2}$ is around 0.5 .

The energy dependence of the quadruple term $a_{4}$ is shown in Fig. 9(c). Again we observe rather similar results for the DPE and $(e, 2 e)$ experiment with $E_{p}=25 \mathrm{eV}$. We conclude that the first visual impression of a transition between a $U$ and a $\mathrm{V}$ shape can be described by the simple functional for the profile presented in Eq. (7). The surprising result is a very similar energy dependence of the parameters $a_{2}$ and $a_{4}$. If we assume that the increase in the term $a_{0}$ for energies above $8 \mathrm{eV}$ is, indeed, mainly due to random events for the DPE data then all three parameters for both experiments are numerically close.

Let us now discuss the blue data points. They come from an $(e, 2 e)$ experiment in which the primary energy has been increased by 4 to $29 \mathrm{eV}$. The constant term $a_{0}$ behaves very much like for the $(e, 2 e)$ result with $E_{p}=25 \mathrm{eV}$. Very different though is the behavior of the quadratic term $a_{2}$ which does not go towards zero upon approaching an energy of $10 \mathrm{eV}$ as for the other two data sets. Compared to the data with $E_{p}=25 \mathrm{eV}$ the $a_{2}$ values near zero occur at 2-eV higher energies. This is half of the primary energy increase which means that the same initial state is probed. The quadruple term $a_{4}$ for $E_{p} \mathrm{eV}$ increases dramatically once the energy exceeds $11 \mathrm{eV}$. Again this occurs for an energy which is $2 \mathrm{eV}$ 


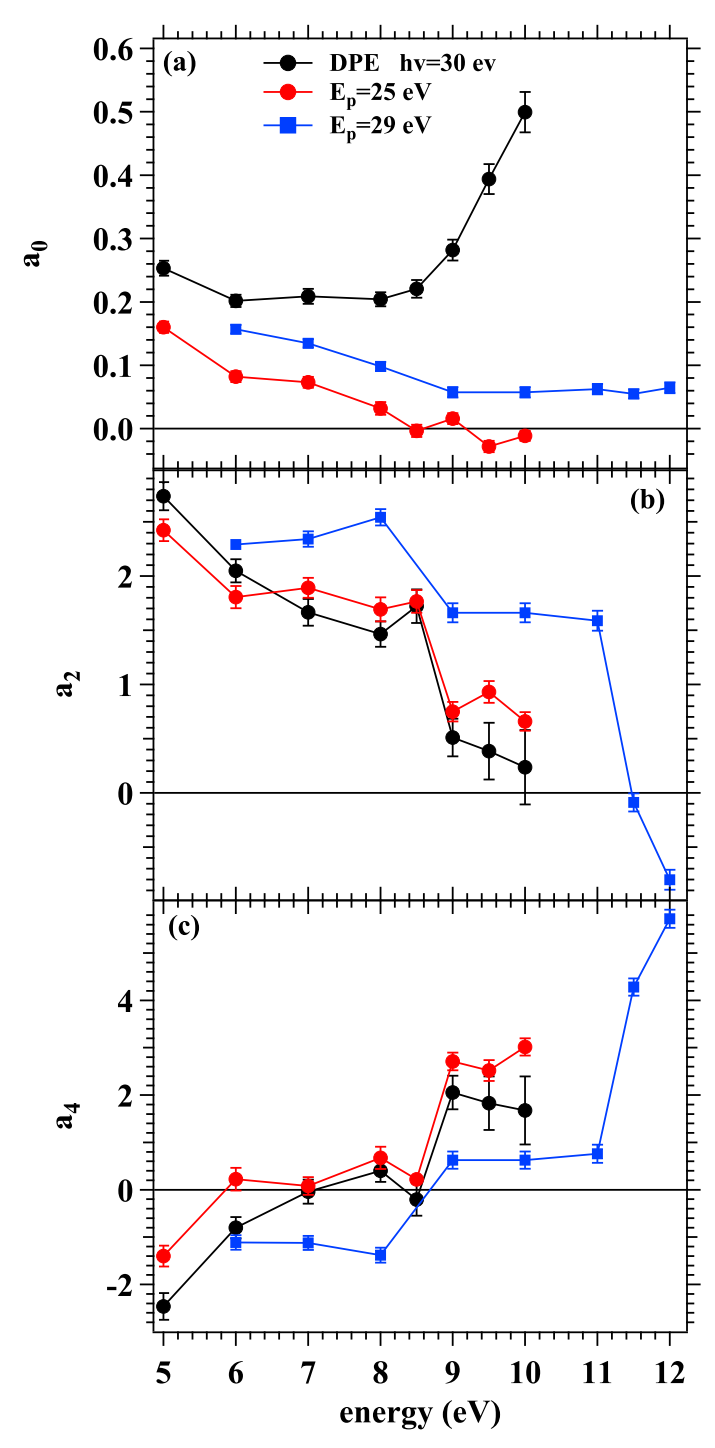

FIG. 9. We summarize the result of the fitting analysis in which the fit parameters have been normalized, see the text. In panel (a) we show the result for $a_{0}$, whereas (b) presents the result of the term $a_{2}$. In (c) the result for $a_{4}$ is shown. The black and red data points refer to the DPE and $(e, 2 e)$ experiment which are kinematically equivalent. The blue $(e, 2 e)$ data points stem from an experiment with $E_{p}=29 \mathrm{eV}$.

higher than for the experiment with $E_{p}=25 \mathrm{eV}$ reflecting the relevance of the initial state. From the behavior of the fit parameters for $E_{p}=29 \mathrm{eV}$ we expect a transition from a V to a $\mathrm{U}$ shape to occur at higher energies compared to the DPE and $(e, 2 e)$ experiment with $E_{p}=25 \mathrm{eV}$. This is demonstrated by the momentum profiles for $E_{p}=29 \mathrm{eV}$ as presented in the Supplemental Material [43].

Obviously the kinetic energy of the outgoing electrons alone does not determine the momentum profile, but the involved band states play an important role.

Let us recall the basics of the theoretical description. The electron-electron interaction was cast into the correlation factor which is a function of the distance and momentum difference of the outgoing electrons. Regardless of the primary energy, the correlated final state remains the same if the energies and emission angles of the outgoing electrons are the same. This applies to $(e, 2 e)$ and DPE. The profiles for the $(e, 2 e)$ experiments with $E_{p}=25$ and $29 \mathrm{eV}$ are different as evidenced by their fit parameters, see Fig. 9. Clearly it is the difference in the initial state which leads to a different matrix element for the transition. The initial state in DPE consists of two valence electrons, and the transition is mediated by the dipole operator. The key message we want to relay is that our results support the notion that $(e, 2 e)$ and DPE probe essentially the initial-state correlation.

Our description of the profiles is by no means a trivial observation. There is no theoretical work which suggests that the use of a three-parameter polynomial is appropriate. Furthermore, it is not expected that the same parameters should describe the DPE and $(e, 2 e)$ line shape equally well. Finally, we are not able to explain the reason for the transition from $\mathrm{U}$ to $\mathrm{V}$ shape. These issues need clarification by a more detailed theory.

\section{SUMMARY}

We have compared the pair emission process from a $\mathrm{Cu}(111)$ surface due electron impact and photon absorption. The emission geometry was kept constant in both studies. The photon and primary electron energy was selected such that both experiments were kinematically equivalent. The analysis of the 2D-energy distributions reveals a very different behavior for DPE compared to $(e, 2 e)$. Although there exist sharp features in $(e, 2 e)$ most notably the contribution from the Shockley surface state, this is not the case for DPE where the intensity changes smoothly. This can be largely explained by the underlying initial states. In $(e, 2 e)$ a single-particle band structure is needed whereas DPE demands a self-convolution. This has the net effect that the spectral weight for Shockley pair emission is more than three orders of magnitude smaller than for a pair of $3 d$ electrons.

The momentum distributions of DPE and $(e, 2 e)$ display a close similarity despite the large difference in the microscopic processes leading to pair emission. There is a distinctive energy dependence from a $\mathrm{U}$ to a $\mathrm{V}$ shape of the profiles. These can be empirically described by a polynomial with three parameters. The numerical values of the parameter are almost the same for both pathways. Additional $(e, 2 e)$ measurements with a different primary electron energy reveal the importance of the initial state on the profiles. This is an experimental confirmation that electron pair emission essentially probes the correlation in the initial state.

\section{ACKNOWLEDGMENTS}

We thank F. Giebels, H. Gollisch, and R. Feder [44] for communicating to us the theoretical band-structure data and enlightening discussions on the theory of pair emission. We thank the HZB for the allocation of the synchrotron beamtime at the BESSY II storage ring and F. Schäfers for support at the beamline. 
[1] J. Berakdar, H. Gollisch, and R. Feder, Solid State Commun. 112, 587 (1999).

[2] N. Fominykh, J. Berakdar, J. Henk, and P. Bruno, Phys. Rev. Lett. 89, 086402 (2002).

[3] F. O. Schumann, J. Kirschner, and J. Berakdar, Phys. Rev. Lett. 95, 117601 (2005).

[4] F. O. Schumann, C. Winkler, G. Kerherve, and J. Kirschner, Phys. Rev. B 73, 041404(R) (2006).

[5] F. O. Schumann, C. Winkler, and J. Kirschner, Phys. Rev. Lett. 98, 257604 (2007).

[6] F. O. Schumann, C. Winkler, and J. Kirschner, New J. Phys. 9, 372 (2007).

[7] M. Hattass et al., Phys. Rev. B 77, 165432 (2008).

[8] F. O. Schumann, N. Fominykh, C. Winkler, J. Kirschner, and J. Berakdar, Phys. Rev. B 77, 235434 (2008).

[9] F. O. Schumann, C. Winkler, J. Kirschner, F. Giebels, H. Gollisch, and R. Feder, Phys. Rev. Lett. 104, 087602 (2010).

[10] F. Giebels, H. Gollisch, R. Feder, F. O. Schumann, C. Winkler, and J. Kirschner, Phys. Rev. B 84, 165421 (2011).

[11] G. A. Fiete and E. J. Heller, Rev. Mod. Phys. 75, 933 (2003).

[12] P. O. Gartland and B. J. Slagsvold, Phys. Rev. B 12, 4047 (1975).

[13] P. Heimann, H. Neddermeyer, and H. F. Roloff, J. Phys. C 10, L17 (1977).

[14] J. A. Knapp, F. J. Himpsel, and D. E. Eastman, Phys. Rev. B 19, 4952 (1979).

[15] S. G. Louie, P. Thiry, R. Pinchaux, Y. Petroff, D. Chandesris, and J. Lecante, Phys. Rev. Lett. 44, 549 (1980).

[16] S. D. Kevan, Phys. Rev. Lett. 50, 526 (1983).

[17] P. Aebi, J. Osterwalder, R. Fasel, D. Naumovic, and L. Schlapbach, Surf. Sci. 307-309, 917 (1994).

[18] F. Reinert, G. Nicolay, S. Schmidt, D. Ehm, and S. Hüfner, Phys. Rev. B 63, 115415 (2001).

[19] H. Gollisch, N. v. Schwartzenberg, and R. Feder, Phys. Rev. B 74, 075407 (2006).

[20] F. Giebels, H. Gollisch, and R. Feder, J. Phys.: Condens. Matter 21, 355002 (2009).

[21] N. Fominykh and J. Berakdar, J. Electron. Spectrosc. Relat. Phenom. 161, 125 (2007).

[22] N. Fominykh, J. Henk, J. Berakdar, and P. Bruno, Surf. Sci. 507, 229 (2002).
[23] F. O. Schumann, R. S. Dhaka, G. A. van Riessen, Z. Wei, and J. Kirschner, Phys. Rev. B 84, 125106 (2011).

[24] F. O. Schumann, C. Winkler, and J. Kirschner, Phys. Rev. B 88, 085129 (2013).

[25] M. Huth, C.-T. Chiang, A. Trützschler, F. O. Schumann, J. Kirschner, and W. Widdra, Appl. Phys. Lett. 104, 061602 (2014).

[26] A. Trützschler, M. Huth, C.-T. Chiang, R. Kamrla, F. O. Schumann, J. Kirschner, and W. Widdra, Phys. Rev. Lett. 118, 136401 (2017).

[27] C.-T. Chiang, A. Trützschler, M. Huth, R. Kamrla, F. O. Schumann, and W. Widdra, Prog. Surf. Sci. 95, 100572 (2020).

[28] M. Munoz-Navia, C. Winkler, R. Patel, M. Birke, F. O. Schumann, and J. Kirscher, J. Phys.: Condens. Matter 21, 355003 (2009).

[29] E. Wigner and F. Seitz, Phys. Rev. 43, 804 (1933).

[30] J. C. Slater, Rev. Mod. Phys. 6, 209 (1934).

[31] W. Kohn, Rev. Mod. Phys. 71, 1253 (1999).

[32] F. Schäfers, W. Peatman, A. Eyers, C. Heckenkamp, G. Schönhense, and U. Heinzmann, Rev. Sci. Instrum. 57, 1032 (1986).

[33] J. Berakdar and H. Klar, Phys. Rev. Lett. 69, 1175 (1992).

[34] J. Viefhaus et al., Phys. Rev. Lett. 77, 3975 (1996).

[35] R. Feder, H. Gollisch, D. Meinert, T. Scheunemann, O. M. Artamonov, S. N. Samarin, and J. Kirschner, Phys. Rev. B 58, 16418 (1998).

[36] J. Berakdar, Phys. Rev. B 58, 9808 (1998).

[37] F. O. Schumann, Y. Aliaev, I. Kostanovskiy, and J. Kirschner, Phys. Rev. B 101, 115104 (2020).

[38] H. Gollisch, X. Yi, T. Scheunemann, and R. Feder, J. Phys.: Condens. Matter 11, 9555 (1999).

[39] R. Feder and H. Gollisch, Solid State Commun. 119, 625 (2001).

[40] U. Rücker, H. Gollisch, and R. Feder, Phys. Rev. B 72, 214424 (2005).

[41] N. Fominykh, J. Henk, J. Berakdar, P. Bruno, H. Gollisch, and R. Feder, Solid State Commun. 113, 665 (2000).

[42] J. L. Powell and B. Crasemann, Quantum Mechanics (Dover, Mineola, New York, 2015).

[43] See Supplemental Material at https://link.aps.org/supplemental/ 10.1103/PhysRevB.103.085435 for more details.

[44] The theoretical band structure data required to make these plots where supplied to us by F. Giebels, H. Gollisch, and R. Feder, Details of the approach can be found at www.flapw.de. 